\title{
Periodontal ligament stem cells in regenerative dentistry for periodontal tissues
}

\begin{abstract}
The ideal periodontal treatment is regenerating functional periodontal tissues destroyed by severe periodontitis. The tissue engineering triad includes stem cells, biological signals, and cell-seeded scaffold that are considered to be essential for tissue regeneration. Periodontal ligament (PDL) tissue contains a stem cell population, periodontal ligament stem cells (PDLSC), that is derived the neural crest tissue. PDLSC demonstrate high proliferative capacity and multipotency that make them a highly promising stem cell population for use in the regeneration of damaged periodontal tissues. Here, we review the current understanding of the features and functions of PDLSC.
\end{abstract}

Keywords: periodontal ligament, regeneration, stem cells, periodontal tissues, biological signals
Volume I Issue 3 - 2016

\author{
Atsushi Tomokiyo,' Naohisa Wada, ${ }^{2}$ Sayuri \\ Hamano,' Daigaku Hasegawa,' Hideki Sugii, ${ }^{3}$ \\ Shinichirou Yoshida,' Hidefumi Maeda' \\ 'Department of Endodontology and Operative Dentistry, \\ Kyushu University, Japan \\ 2Division of Oral Clinic, Kyushu University Hospital, Japan \\ ${ }^{3}$ Center for Craniofacial Molecular Biology, University of \\ Southern California, USA
}

\begin{abstract}
Correspondence: Atsushi Tomokiyo, Department of Endodontology and Operative Dentistry, Faculty of Denta Science, Kyushu University, Maidashi 3-I-I, Higashi-ku, Fukuokashi, Fukuoka, Japan, Tel +8I-92-642-6432, Email tomokiyo@dent.kyushu-u.ac.jp
\end{abstract}

Received: July 0I, 2016 | Published: July 18, 2016

\section{Introduction}

The periodontal ligament (PDL), a fibrous connective tissue structure, plays crucial roles in tooth anchorage, sensation, and facilitating nutrient supply to surrounding tissues. ${ }^{1}$ Periodontitis is a chronic inflammatory condition of the periodontium and around $50 \%$ of adults suffer from its moderate and severe generalized form ${ }^{2}$ Severe generalized form of periodontitis induces the destruction of PDL tissues including the loss of collagen fiber attachment between alveolar bones to the cementum surface and the progression of alveolar bone resorption, which is a principal cause for tooth loss. ${ }^{3}$ Therefore, the regeneration of PDL tissues damaged by advanced periodontitis would be important to maintain healthy teeth for a lifetime. Stem cells are self-sustaining by replicating themselves and show the potential to differentiate into various cell types. Many researchers believe that these features of stem cells may offer benefits in the field of cellbased regenerative therapy. PDL stem cells (PDLSC) are a kind of dental stem cells identified in PDL tissues and originate from the neural crest tissue. They also have the potential to differentiate into a variety of PDL cells including fibroblasts, osteoblasts, cementoblasts, enthothelial cells, neural cells. ${ }^{4,5}$ The aim of this review is to summarize the key topics of PDLSC including their properties, characteristics, current research, and future potential for PDLSC-based regenerative periodontal therapies.

\section{Characteristic of periodontal ligament stem cells}

The stem/progenitor cell populations have been revealed to be derived from endosteal spaces that are enriched immmature cells. ${ }^{6}$ These cells have the potential to differentiate into multipule PDL cell including fibroblasts, osteoblasts, and cementoblasts that are principal for maintainance and homeostasis of PDL tissues. ${ }^{7}$ Seo et al. ${ }^{8}$ first isolated PDLSC from the PDL tissue of extracted human third molar teeth. ${ }^{8}$ Many types of MSC-related cell surface markers such as CD10,
CD13, CD26, CD29, CD44, CD71, CD73, CD90, CD105, CD106, CD146, CD166, CD349, STRO-1, STRO-3, and TNAP/MSCA-1 have been identified in PDLSC. ${ }^{9}$ PDLSC formed mineralized nodules with the increase of bone-related gene expression when they were cultured in osteogenic medium. ${ }^{8}$ Additionally, PDLSC injected into calvarial defect model mice demonstrated the new bone formation within the defects, ${ }^{10}$ suggesting that PDLSC have the capacity to differentiate into osteoblasts. PDLSC was also induced to differentiate into chondrocytes and adipocytes upon exposure to specific differentiation media. ${ }^{8,11}$ PDLSC can differentiate into endothelial cells that generated capillary-like sprouts with lumens in vitro. ${ }^{12}$ Imoprtantly, LY294002, a PI3K inhibitor, completely suppressed the blood vessel generation and significnaly inhibited proliferation and endothelial cell-related marker expression in PDLSC, suggesting that PDLSC requires PI3K activation in their endothelial cell differentiation. ${ }^{12}$ PDLSC have the potential to form neurosheres, three-dimensional floating cell clusters containing neural stem/progenitor cells..$^{13}$ Moreover, recent studies have demonstrated the generation of retial cells from PDLSC by the formation of neurospheres and inhibition of Wnt and BMP signalings. ${ }^{14}$ Our recent study demonstrated that semaphorin $3 \mathrm{~A}$ (Sema3A) plays a crucial role in preserving stem cell properties of PDLSCs; the expression level of Sema3A was stronger in multipotent human PDL cell lines compared with low-differentiation potential lines, and Sema3A-overexpressing low-differentiation potential PDL clones increased ESC- and MSC-related marker expression and enhanced capacity to differentiate into osteoblasts and adipocytes. ${ }^{15}$ Additionally, PDLSC exert immunomodulatory effects through their low immunogenicity by the absence of HLA-II DR and T cell costimulatory molecules, suppression of proliferation, differentiation, and migration in $\mathrm{B}$ cells by cell-to-cell contact, and inhibition of peripheral blood mononuclear cell proliferation as a result of cellcycle inhibition rather than of apoptosis..$^{16,17}$ 


\section{Potential of PDLSC to regenerate periodontal struc- tures}

The regenerative effects of several biological signals on PDLSC have been demonstrated. Transforming growth factor beta 1 (TGF- $\beta 1$ ) induced collagen type I and $\alpha$-smooth muscle actin ( $\alpha$-SMA) gene transcription and $\alpha$-SMA protein expression in PDLSC, however differentiated PDL cells revealed no change in their expression. ${ }^{18}$ This result indicates the important roles of TGF- $\beta 1$ in collagen and $\alpha$-SMA production of predominantly immature PDL cells. Connective tissue growth factor (CTGF) promoted collagen type I and III, biglycan, and periostin protein synthesis and mineralized nodule formation in PDLSC. ${ }^{19}$ Our recent study also demonstrated that CTGF upregulated proliferation, migration, osteogenic gene expression, and mineralization in PDLSC. ${ }^{20}$ Additionally, a variety of studies have tried to regenerate PDL tissues using PDLSC in vivo. Collagen is the most abundant protein in the body and mainly makes up the fibrous elements of PDL tissues. ${ }^{21}$ The subcutaneously implanted PDLSC with a hyaluronic acid-based scaffold demonstrated a greater amount and density of collagen synthesis than adipose tissue-derived stem cells. ${ }^{22}$ Subcutaneous injection of PDLSC with hydroxyapatite or beta-tricalcium phosphate scaffolds resulted in the generation of cementum- and PDL-like structures adjacent to the surfaces of scaffolds. ${ }^{8}$ Dentin blocks wrapped in the PDLSC sheet showed the potential to develop well-organized PDL tissues with perpendicular and oblique orientation of collagen fibers on the dentin surfaces. ${ }^{23}$ In another study, PDLSC transplanted into fenestration defects with fiber-guiding scaffolds produced by the rapid prototyping technique induced the regeneration of PDL-like tissues that showed angulations similar to healthy and mature ligamentous tissues. ${ }^{24}$ Moreover, injection of autologous PDLSC with bone grafting materials into the intrabony defects of deep periodontal pockets in 3 periodontitis patients significantly improved the value of clinical index including probing depth, gingival recession, and attachment level, and attachment gain, which persisted for up to 72 months. ${ }^{25}$

\section{Future perspectives}

As reviewed in this article, it has been revealed that PDLSC participate in the development and regeneration of PDL tissues. Adult PDL tissues contain stem cells; however there still is a difficulty in isolating a pure PDLSC population because of the rarity of PDLSC in vivo and their limited replicative capacity. Induced pluripotent stem cells (iPSC) are especially promising cell sources for tissue engineering because iPSC reveal embryonic stem cell-like properties including high multi-differentiation and self-renewal capabilities. ${ }^{26,27}$ PDLSC is originated from neural crest cells during development and our and other studies have demonstrated the successful generation of neural crest-like cells (NCLC) from iPSC. ${ }^{28-33}$ Importantly, subcutaneously transplanted iPSC-derived NCLC revealed no capacity to form tumors. Since iPSC-derived NCLC would work out the rarity and limited replicative capacity of PDLSC and decrease the risk of iPSC-related tumor formation, it is therefore possible that iPSC-derived NCLC could become the alternatives of PDLSC and the prospective cells for the achievement of complete PDL tissue regeneration.

\section{Acknowledgements}

None.

\section{Conflict of interest}

The author declares no conflict of interest.

\section{References}

1. Carnes DL, Maeder CL, Graves DT. Cells with osteoblastic phenotypes can be explanted from human gingiva and periodontal ligament. $J$ Periodont. 1997;68(7):701-707.

2. Pejcic A, Kojovic D, Mirkovic D, et al. Stem cells for periodontal regeneration. Balkan J Med Genet. 2013;16(1):7-12.

3. Aida J, Ando Y, Akhter R, et al. Reasons for permanent tooth extractions in Japan. J Epidemiol. 2006;16(5):214-219.

4. Tomokiyo A, Maeda H, Fujii S, et al. Development of a multipotent clonal human periodontal ligament cell line. Differentiation. 2008;76(4):337-347.

5. Teramatsu Y, Maeda H, Sugii H, et al. Expression and effects of epidermal growth factor on human periodontal ligament cells. Cell Tissue Res. 2014;357(3):633-643.

6. Melcher AH. On the repair potential of periodontal tissues. $J$ Periodontol. 1976;47(5):256-260

7. McCulloch CA, Nemeth E, Lowenberg B, et al. Paravascular cells in endosteal spaces of alveolar bone contribute to periodontal ligament cell populations. Anat Rec. 1987;219(3):233-242.

8. Seo BM, Miura M, Gronthos S, et al. Investigation of multipotent postnatal stem cells from human periodontal ligament. Lancet. 2004;364(9429):149-155.

9. Tomokiyo A, Wada N, Maeda H. Contribution of stem cells to dental tissue regeneration: isolation, function, and application. Frontiers in Stem Cell and Regenerative Medicine Research. 2016;2:3-38.

10. Moshaverinia A, Chen C, Xu X, et al. Bone regeneration potential of stem cells derived from periodontal ligament or gingival tissue sources encapsulated in RGD-modified alginate scaffold. Tissue Eng Part A. 2014;20(3-4):611-621.

11. Choi S, Cho TJ, Kwon SK, et al. Chondrogenesis of periodontal ligament stem cells by transforming growth factor-beta 3 and bone morphogenetic protein-6 in a normal healthy impacted third molar. Int $J$ Oral Sci. 2013;5(1):7-13.

12. Okubo N, Ishisaki A, Iizuka T, et al. Vascular cell-like potential of undifferentiated ligament fibroblasts to construct vascular cell-specific marker-positive blood vessel structures in a PI3K activation-dependent manner. J Vasc Res. 2010;47(5):369-383.

13. Techawattanawisal W, Nakahama K, Komaki M, et al. Isolation of multipotent stem cells from adult rat periodontal ligament by neurosphere-forming culture system. Biochem Biophys Res Commun. 2007;357(4):917-923.

14. Huang L, Liang J, Geng Y, et al. Directing adult human periodontal ligament-derived stem cells to retinal fate. Invest Ophthalmol Vis Sci. 2013;54(6):3965-3974.

15. Wada N, Maeda H, Hasegawa D, et al. Semaphorin $3 A$ induces mesenchymal-stem-like properties in human periodontal ligament cells. Stem Cells Dev. 2014;23(18):2225-2236.

16. Wada N, Menicanin D, Shi S, et al. Immunomodulatory properties of human periodontal ligament stem cells. J Cell Physiol. 2009;219(3):667-676

17. Zhu W, Liang M. Periodontal ligament stem cells: current status, concerns, and future prospects. Stem Cells Int. 2015;2015(2015):11. 
18. Fujii S, Maeda H, Tomokiyo A, et al. Effects of TGF-beta1 on the proliferation and differentiation of human periodontal ligament cells and a human periodontal ligament stem/progenitor cell line. Cell Tissue Res. 2010;342(2):233-242.

19. Dangaria SJ, Ito Y, Walker C, et al. Extracellular matrix-mediated differentiation of periodontal progenitor cells. Differentiation. 2009;78(2-3):79-90.

20. Yuda A, Maeda H, Fujii S, et al. Effect of CTGF/CCN2 on osteo/cementoblastic and fibroblastic differentiation of a human periodontal ligament stem/progenitor cell line. J Cell Physiol. 2015;230(1):150-159.

21. Sodek J, Brunette DM, Feng J, et al. Collagen synthesis is a major component of protein synthesis in the periodontal ligament in various species. Arch Oral Biol. 1977;22(12):647-653.

22. Jung IH, Park JC, Kim JC, et al. Novel application of human periodontal ligament stem cells and water-soluble chitin for collagen tissue regeneration: in vitro and in vivo investigations. Tissue Eng Part A. 2012;18(5-6):643-653.

23. Ji K, Liu Y, Lu W, et al. Periodontal tissue engineering with stem cells from the periodontal ligament of human retained deciduous teeth. $J$ Periodontal Res. 2013;48(1):105-116.

24. Park CH, Rios HF, Taut AD, et al. Image-based, fiber guiding scaffolds: a platform for regenerating tissue interfaces. Tissue Eng Part C Methods. 2014;20(7):533-542.

25. Feng F, Akiyama K, Liu Y, et al. Utility of PDL progenitors for in vivo tissue regeneration: a report of 3 cases. Oral Dis. 2010;16(1):20-28.
26. Takahashi K, Yamanaka S. Induction of pluripotent stem cells from mouse embryonic and adult fibroblast cultures by defined factors. Cell. 2006;126(4):663-676.

27. Yu J, Vodyanik MA, Smuga Otto K, et al. Induced pluripotent stem cell lines derived from human somatic cells. Science. 2007;318(5858):1917-1920.

28. Okawa T, Kamiya H, Himeno T, et al. Transplantation of neural crestlike cells derived from induced pluripotent stem cells improves diabetic polyneuropathy in mice. Cell Transplant. 2013;22(10):1767-1783.

29. Liu Q, Spusta SC, Mi R, et al. Human neural crest stem cells derived from human ESCs and induced pluripotent stem cells: induction, maintenance, and differentiation into functional schwann cells. Stem Cells Transl Med. 2012;1(4):266-278.

30. Saadai P, Wang A, Nout YS, et al. Human induced pluripotent stem cell-derived neural crest stem cells integrate into the injured spinal cord in the fetal lamb model of myelomeningocele. J Pediatr Surg. 2013;48(1):158-163.

31. Xu W, Wang Y, Liu E, et al. Human iPSC-derived neural crest stem cells promote tendon repair in a rat patellar tendon window defect model. Tissue Eng Part A. 2013;19(21-22):2439-2451.

32. Otsu K, Kishigami R, Oikawa Sasaki A, et al. Differentiation of induced pluripotent stem cells into dental mesenchymal cells. Stem Cells Dev. 2012;21(7):1156-1164.

33. Tomokiyo A, Hynes $\mathrm{K}, \mathrm{Ng}$ J, et al. Generation of neural crest-like cells from human periodontal ligament cell-derived induced pluripotent stem cells. J Cell Physiol. 2017;232(2):402-416. 